\title{
Nurses' Occupational and Medical Risks Factors of Leaving the Profession in Nursing Homes
}

\author{
Carole Pélissier ${ }^{1,2, *}$, Barbara Charbotel ${ }^{3,4}$, Jean Baptiste Fassier ${ }^{3,5}$, Emmanuel Fort ${ }^{3}$ (D) \\ and Luc Fontana 1,2 \\ 1 Unité Mixte de Recherche Épidémiologique et de Surveillance Transport Travail \\ Environnement (UMRESTTE, UMR_T9405), Institut français des sciences et technologies des transports, \\ de l'aménagement et des réseaux (IFSTTAR), Université de Lyon, Université Lyon 1, Université de St \\ Etienne, 42055 Saint-Etienne, France; luc.fontana@chu-st-etienne.fr \\ 2 Service de Santé au Travail et Pathologies Professionnelles, CHU de Saint-Etienne, 42055 Saint-Etienne, \\ France \\ 3 Unité Mixte de Recherche Épidémiologique et de Surveillance Transport Travail \\ Environnement (UMRESTTE, UMR_T9405), Institut français des sciences et technologies des transports, \\ de l'aménagement et des réseaux (IFSTTAR), Université de Lyon, Université Lyon 1, F-69373 Lyon, France; \\ barbara.charbotel-coing-boyat@univ-lyon1.fr (B.C.); jean-baptiste.fassier@chu-lyon.fr (J.B.F.); \\ emmanuel.fort@univ-lyon1.fr (E.F.) \\ 4 Hospices Civils de Lyon, Centre Hospitalier Lyon Sud, Service de Pathologies Professionnelles, F-69495 \\ Pierre Bénite, France \\ 5 Hospices Civils de Lyon, Service de Médecine et Santé au Travail, 69002 Lyon, France \\ * Correspondence: Pelissier.carole.chu@gmail.com; Tel.: +33-(0)4-7782-8325; Fax: +33-(0)4-7782-8139
}

Received: 8 August 2018; Accepted: 23 August 2018; Published: 27 August 2018

\begin{abstract}
This study aimed to evaluate the association between intention to leave work, and working conditions and health status among female care-staff in nursing homes. A multicenter cross-sectional study included female care-staff in 105 nursing homes for the elderly. We used validated questionnaires to assess occupational, psychosocial and medical data in a multicenter transverse study. Univariate analysis on $\mathrm{chi}^{2}$ test was performed with stratification according to job (nurse, nursing assistant), and variables found to be significant on each dimension were included on multivariate models. 1428 nursing assistants and 342 registered nurses were included. 391 nursing assistants and 85 registered nurses intended to leave their work with the elderly. The registered nurses' intention to leave was associated with deteriorated care-team or residents relations, and with perceived elevated hardship due to the proximity of residents' death. The nursing assistants' intention to leave was associated with deteriorated management relation, with job insecurity and elevated hardship due to the residents' intellectual deterioration. Impaired physical or psychological health status also correlated with this intention. Policy to reduce voluntary turnover of care-staff in nursing homes for the elderly could be based on multifactorial management, acting on work organization and reducing psychosocial stress.
\end{abstract}

Keywords: care workers' intention to leave; nursing homes for the elderly; psychosocial factors; musculoskeletal complaints; impaired well-being

\section{Introduction}

High rates of staff turnover in nursing homes are not a recent phenomenon, especially among those who work most closely with the residents: registered nurses and nursing assistants. Nursing homes for the elderly differ in functioning from hospitals in that they have lower level of medical presence. They are residential establishments caring for increasingly elderly, dependent persons with 
multiple comorbidities. Care is given by nursing assistants with various kinds of initial training and by registered nurses. Registered nurses usually ensure technical care and coordinate the work of the nursing assistants. As well as catering and accompaniment, nursing assistants are in charge of hygiene, comfort and preventive and curative care and are under the supervision of a nurse. About $17.5 \%$ of new nurses leave their first job within one year of starting their jobs [1]. The proportion of nurses considering or intending to leave the profession varied from $4 \%$ to $54 \%$ [2]. The cost of turnover from the organizational perspective includes replacement costs (including training), lost productivity, lost quality and lowered morale [3,4]. A previous study argued that these costs may well be reflected in the quality of care that residents receive $[3,5]$. Three broad categories of factors that influence turnover have been identified: general economic, work-related and personal factors [3]. Registered nurses and nursing assistants in nursing homes are subject to strong mental and physical demands (e.g., lifting and carrying, work schedule), and frequently describe their working environment as distressing [6]. The physical and mental deterioration of elderly patients and the proximity to death were more often perceived as a source of hardship by registered nurses and nursing assistants. Registered nurses and nursing assistants are more often confronted by aggressive patients than in hospitals or facilities for the disabled [7-9]. Registered nurses who had experienced burnout or stress were more likely to intend to leave the profession $[2,10]$. Fochsen et al. have focused on the relationship between musculoskeletal disorders and intent to leave the profession in staff of nursing-homes for the elderly [11]. Several studies underlined an association between impaired mental well-being in nursing home care workers and psychosocial factors in health caregivers, but there has been less research on the association between impaired mental well-being in nursing home care workers and health caregivers' intention to leave has hardly been studied [12-15]. Understanding why health care workers abandon their current employer and/or their job in the nursing profession is important in retention of health providers. The objective of the present study was to analyze occupational and medical factors associated with the intention to leave work with the elderly, comparing between nursing assistants and registered nurses, considering the differences in their tasks.

\section{Materials and Methods}

\subsection{Design}

The study was designed as a cross-sectional questionnaire survey design.

\subsection{Participants}

The target population of the survey was female employees working in with elderly patients in nursing homes in the Rhône-Alpes Region of France. The occupational physicians of the Region were asked to participate in the survey by the Regional Department of Businesses, Competition, Consumption, Work and Employment (DIRECCTE), a state business consultancy. Volunteer occupational physicians filled out a working conditions assessment questionnaire, and asked all employees meeting the inclusion criteria in the nursing homes which they oversaw to take part. Only employees who had been working with the elderly for at least 6 months on at least a half-time basis were included. The occupational physician handed over the self-administered questionnaire to subjects included in the study and collected data on working conditions and health status in nursing staff. The number of subjects who refused to participate was anonymously collected by the occupational physicians.

\subsection{Data Collection}

Between October 2009 and September 2010, data were collected by self-administered questionnaires during periodic follow-up medical examinations or during company visits. These self-administered questionnaires comprised personal characteristics (age, gender, family status, number of children), health characteristics and work-related characteristics and used four 
questionnaires measuring Job related hardship, musculoskeletal complaints, impaired mental well-being, and psychosocial demands [16-21].

\subsection{Measures}

Job related hardship: The questionnaire included visual analog hardship scales $(1=$ no hardship, to $10=$ great hardship) related to: premises (disrepair, stairs, clutter), lifting and carrying the elderly, patients' mental and physical deterioration, and proximity of death [16-18]. The choice of these particular categories was based on the literature [21]. Hardship scores were categorized as slight $(\leq 3)$, moderate (4-7) or severe hardship $(\geq 8)$.

Musculoskeletal complaints: Musculoskeletal complaints were assessed on the Nordic Musculoskeletal Questionnaire, comprising multiple choice questions for each body part (such as upper limbs, lower limbs, neck and back): "During the last 12 months, have you had trouble (such as ache, pain, discomfort)?". The Nordic Musculoskeletal Questionnaire is a standardized questionnaire with reliability and validity moderate to high, which makes is a good instrument for epidemiological survey into musculoskeletal disorders and complaints [19].

Impaired mental well-being: Developed as a screening tool to detect the workers likely to have or to risk of developing psychiatry disorders, the General Health Questionnaire (GHQ) is a measure of the common mental health problems, domains of depression, anxiety, somatic symptoms and social withdrawal. It's the 12-item version (GHQ-12) was used. Respondents had to indicate how frequently they had recently experienced the various symptoms listed. Each item was rated on a 4-point scale, with weights from 0 to 3 according to Likert-like type scale $(0=$ less than usual, $1=$ no more than usual, $2=$ rather more than usual, and $3=$ much more than usual) [20]. Scores higher than 12 were considered as indicating impaired mental wellbeing [22,23].

Psychosocial demands: Psychosocial demands at work were assessed on the effort-reward imbalance (ERI) model using the Siegrist questionnaire. In this model, chronic work-related stress is identified as non-reciprocity or imbalance between high efforts spent and low rewards received. Conversely, positive emotions evoked by appropriate social rewards promote well-being, health and survival [16]. This questionnaire has three scales: two measuring the extrinsic components of 'effort' (6 items) and 'reward' (11 items covering earnings, esteem and job security) and one measuring the intrinsic component of 'over-commitment' (six items) [16,17]. Effort was measured either by six items on the demanding aspect of the work environment (three measuring quantitative load, one qualitative load, one increase in total load over time, and one physical load), rated as $1=$ does not apply, $2=$ does apply but subject does not consider herself distressed, $3=$ does apply and subject considers herself somewhat distressed, $4=$ does apply and subject considers herself distressed, or $5=$ does apply and subject considers herself very distressed. A sum score of these ratings was totalled, as documented in several studies [16,17]. According to the ERI model, extrinsic and intrinsic effort scores are directly proportional to effort, whereas the rewards score is inversely proportional to reward. ERI was measured by calculating the ratio between the extrinsic effort index $(\mathrm{E})$ and the inverse reward index $(\mathrm{R}): \mathrm{E} /\left(\mathrm{R}^{*} \mathrm{c}\right)$, with $\mathrm{c}$ as a correction factor (c: 6/11); ERI $>1$ indicates a critical condition of high-cost/low-gain, or effort-reward imbalance $[16,17]$. To obtain a more precise view of which rewards were related to which indicators of employee health, three occupational rewards (earnings, esteem and job security) were assessed separately. All three reward scores were dichotomized separately, using the most adverse tercile to indicate low reward: the higher the reward score, the lower the reward [16-18,21,24,25].

\subsection{Ethical Considerations}

Approval by the French Ministry of Health Research (Comité Consultatif pour le Traitement de l'Information en Matière de Recherche dans le Domaine de la Santé, 09.320) was obtained before starting the study. The participants were given an information leaflet explaining the study objectives and participation was voluntary. 


\subsection{Data Analysis}

The continuous quantitative variable of seniority in the establishment was transformed into an ordinal qualitative variable for statistical purposes.

A descriptive step characterized the employee population according to personal characteristics, working conditions, including occupational psychosocial factors, and mental health status. Distinguishing between registered nurses and nursing assistants, associations were sought between the intent to leave work with the elderly and personal factor, occupational factors, psychosocial factors and medical factors. Frequencies were compared on chi-squared tests, with chi-squared trend tests depending on the results of cross-analysis. Ratios of event probabilities per case of intent to leave were studied. As the prevalence of each event was high, odds ratios would not provide a good estimate of relative risk, and a log-linked binomial model was applied, using the PROC GENMOD procedure in the SAS statistical package (version 9.3) with the DIST = BINOMIAL and LINK = LOG options.

Univariate and multivariate analyses were performed stratified on job category (nurse/nursing assistant). The binary response of each case of intent to leave was modeled in two steps: Firstly, all independent variables underwent univariate analysis; secondly, multivariate analyses were performed for each dimension (sociodemographic, occupational, psychosocial, and personal health); variables with $p$-value $\leq 0.1$ were included in a multivariate model on a descending procedure, and variables with $p$-value $<0.05$ were kept in the model. The psychosocial and medical dimension models were adjusted on age. For the psychosocial dimension, the global ERI variable is classically used, but in the present study we sought to investigate the questionnaire sub-dimensions in greater detail, studying the four variables of extrinsic effort and the three types of reward (esteem, earnings, and job security) in the multivariate models. From these four multivariate models, multivariate regression was performed on a descending procedure, with a significance threshold of $5 \%$. In case of non-convergence of PROC GENMOD because the maximum likelihood estimate (MLE) lay on the boundary of the parameter space, the SAS COPY macro was used, which provides a good approximation to the exact maximum likelihood estimates, as well as yielding good estimates of the true population parameters [26].

\section{Results}

\subsection{Socio-Occupational and Medical Data}

1770 women (19.6\% registered nurses, $80.4 \%$ nursing assistants) working in direct contact with the elderly in 105 nursing homes were included. Twenty-nine registered nurses and nine nursing assistants refused to participate, leading to a global participation rate of $98 \%$. Four hundred and seventy-six of the 1808 women (26.3\%), wished to leave their work with the elderly: 391 nursing assistants $(26.8 \%)$ and 85 registered nurses $(24.2 \%)$.

\subsection{Factors Associated with Intention to Leave Work on Univariate Analysis}

Occupational factors: In both nursing assistants and registered nurses, severe hardship related to residents' physical deterioration, to proximity to death, or to lack of equipment was significantly associated with intention to leave. In nursing assistants, weak attachment to the elderly residents, understaffing, and severe hardship related to lifting and carrying residents, to premises, or to residents' intellectual deterioration, were significantly associated with intention to leave (Table 1). In both nursing assistants and registered nurses, work contract, seniority in work with elderly, working time, night work and type of working hours were not associated with intention to leave. 
Table 1. Sociodemographic, occupational, psychosocial and medical factors associated with registered nurses' and nursing assistants' intention to leave work with the elderly, on univariate analysis.

\begin{tabular}{|c|c|c|c|c|c|c|c|c|c|}
\hline \multirow[b]{2}{*}{ Sociodemographic Factors } & & \multicolumn{4}{|c|}{ Nursing Assistants' Intention to Leave $(n=391)$} & \multicolumn{4}{|c|}{ Registered Nurses' Intention to Leave $(\mathrm{N}=85)$} \\
\hline & & $\mathbf{N}$ & $\%$ & PR & {$[95 \% \mathrm{CI}]$} & $\mathbf{N}$ & $\%$ & PR & {$[95 \% \mathrm{CI}]$} \\
\hline \multirow{4}{*}{ Age } & $<30$ years & 118 & 33.9 & $1^{* * * *}$ & 17 & 27.9 & $1^{\nabla}$ & & \\
\hline & $30-39$ years & 97 & 31.0 & 0.91 & $0.74-1.14$ & 24 & 33.3 & 1.19 & $0.71-2.01$ \\
\hline & $40-49$ years & 110 & 24.5 & 0.72 & $0.58-0.90$ & 24 & 25.8 & 0.93 & $0.54-1.57$ \\
\hline & $\geq 50$ years & 65 & 20.5 & 0.60 & $0.46-0.78$ & 20 & 17.2 & 0.62 & $0.35-1.09$ \\
\hline \multirow{3}{*}{ Marital status } & Single & 92 & 32.7 & $1 *$ & 10 & 29.4 & 1 & & \\
\hline & In couple & 237 & 25.4 & 0.77 & $0.64-0.94$ & 62 & 24.8 & 0.84 & $0.48-1.48$ \\
\hline & Separated, divorced, widowed & 58 & 28.6 & 0.87 & $0.66-1.15$ & 13 & 22.8 & 0.77 & $0.38-1.57$ \\
\hline \multirow{2}{*}{ Do you have children? } & Yes & 267 & 25.4 & $1 * *$ & 68 & 24.9 & 1 & & \\
\hline & No & 123 & 33.0 & 1.30 & $1.09-1.55$ & 17 & 24.6 & 0.98 & $0.62-1.57$ \\
\hline Occupational Factors & & $\mathbf{N}$ & $\%$ & PR & {$[95 \% \mathrm{CI}]$} & $\mathbf{N}$ & $\%$ & PR & {$[95 \% \mathrm{CI}]$} \\
\hline \multirow{3}{*}{$\begin{array}{l}\text { Hardship: lifting and } \\
\text { carrying }\end{array}$} & Mild & 36 & 16.4 & $1^{* * * *}$ & 18 & 20.4 & 1 & & \\
\hline & Moderate & 108 & 22.6 & 1.37 & $0.97-1.93$ & 34 & 25.0 & 1.22 & $0.74-2.02$ \\
\hline & Severe & 245 & 34.1 & 2.07 & $1.51-2.85$ & 32 & 28.6 & 1.40 & $0.84-2.31$ \\
\hline \multirow{3}{*}{ Hardship: premises } & Mild & 161 & 11.4 & $1 * *$ & 28 & 20.3 & 1 & & \\
\hline & Moderate & 133 & 30.7 & 1.31 & $1.08-1.59$ & 27 & 26.0 & 1.28 & $0.80-2.03$ \\
\hline & Severe & 245 & 32.7 & 1.40 & $1.13-1.73$ & 30 & 31.60 & 1.56 & $0.99-2.42$ \\
\hline \multirow{3}{*}{ Hardship: equipment } & Mild & 139 & 21.3 & $1^{* * * * *}$ & 32 & 20.0 & $1 *$ & & \\
\hline & Moderate & 143 & 30.7 & 1.44 & $1.18-1.76$ & 27 & 25.5 & 1.27 & $0.81-1.99$ \\
\hline & Severe & 103 & 36.9 & 1.73 & $1.39-2.14$ & 25 & 36.2 & 1.81 & $1.16-2.81$ \\
\hline \multirow{3}{*}{$\begin{array}{l}\text { Hardship: residents' } \\
\text { intellectual deterioration }\end{array}$} & Mild & 54 & 16.8 & $1 * * * *$ & 14 & 15.4 & 1 & & \\
\hline & Moderate & 115 & 25.1 & 1.44 & $1.18-1.76$ & 28 & 21.1 & 1.57 & $0.88-2.80$ \\
\hline & Severe & 218 & 34.4 & 1.73 & $1.40-2.14$ & 43 & 32.6 & 2.11 & $1.24-3.64$ \\
\hline \multirow{3}{*}{$\begin{array}{l}\text { Hardship: residents' physical } \\
\text { deterioration }\end{array}$} & Mild & 46 & 17.1 & $1 * * * *$ & & 13 & 15.7 & $1 *$ & \\
\hline & Moderate & 112 & 24.7 & 1.45 & $1.06-1.96$ & 30 & 23.3 & 1.48 & $0.82-2.68$ \\
\hline & Severe & 230 & 33.1 & 1.94 & $1.46-2.57$ & 42 & 38.8 & 2.09 & $1.20-3.66$ \\
\hline \multirow{3}{*}{ Hardship: proximity to death } & Mild & 90 & 22.4 & $1 * *$ & & 24 & 19.7 & $1 *$ & \\
\hline & Moderate & 128 & 27.0 & 1.21 & $0.95-1.52$ & 32 & 22.7 & 1.15 & $0.72-1.85$ \\
\hline & Severe & 172 & 31.7 & 1.41 & $1.13-1.76$ & 29 & 37.7 & 1.91 & $1.21-3.03$ \\
\hline \multirow{4}{*}{$\begin{array}{l}\text { Do you get attached to the } \\
\text { residents? }\end{array}$} & Very often & 59 & 21.4 & $1 *$ & & 6 & 13.0 & $1^{\nabla}$ & \\
\hline & Often & 235 & 27.5 & 1.29 & $1.00-1.65$ & 52 & 24.9 & 1.91 & $0.87-4.17$ \\
\hline & Seldom & 78 & 31.8 & 1.49 & $1.11-1.99$ & 24 & 34.3 & 2.63 & $1.16-5.93$ \\
\hline & Rarely/never & 19 & 39.6 & 1.85 & $1.22-2.81$ & 3 & 20.00 & 1.53 & $0.44-5.39$ \\
\hline \multirow{2}{*}{ Staffing levels adequate? } & Yes & 58 & 20.1 & $1^{* * *}$ & & 10 & 15.6 & $1^{\nabla}$ & \\
\hline & No & 331 & 29.4 & 1.46 & $1.14-1.87$ & 75 & 27.4 & 1.75 & $0.96-3.19$ \\
\hline
\end{tabular}


Table 1. Cont.

\begin{tabular}{|c|c|c|c|c|c|c|c|c|c|}
\hline \multirow[b]{2}{*}{ Psychosocial Factors } & & \multicolumn{4}{|c|}{ Nursing Assistants' Intention to Leave $(n=391)$} & \multicolumn{4}{|c|}{ Registered Nurses' Intention to Leave $(\mathrm{N}=85)$} \\
\hline & & $\mathbf{N}$ & $\%$ & PR & {$[95 \% \mathrm{CI}]$} & $\mathbf{N}$ & $\%$ & PR & {$[95 \% \mathrm{CI}]$} \\
\hline \multirow{2}{*}{ Victim of verbal aggression } & No & 51 & 17.6 & $1^{* * * *}$ & \multirow[b]{2}{*}{$1.29-2.20$} & 12 & 23.5 & 1 & \multirow[b]{2}{*}{$0.62-1.82$} \\
\hline & Yes & 340 & 29.8 & 1.69 & & 73 & 25.1 & 1.06 & \\
\hline \multirow{2}{*}{ Victim of physical aggression } & No & 137 & 22.2 & $1^{* * * *}$ & \multirow[b]{2}{*}{$1.18-1.69$} & 41 & 23.7 & 1 & \multirow[b]{2}{*}{$0.76-1.59$} \\
\hline & Yes & 254 & 31.3 & 1.41 & & 44 & 26.0 & 1.09 & \\
\hline \multirow[b]{2}{*}{ Relations with team } & Satisfactory/very satisfactory & 308 & 26.5 & $1 \nabla$ & \multirow[b]{2}{*}{$0.98-1.46$} & 60 & 21.1 & $1^{* * *}$ & \multirow[b]{2}{*}{$1.43-3.00$} \\
\hline & Moderately satisfactory/unsatisfactory & 81 & 31.6 & 1.19 & & 25 & 43.9 & 2.07 & \\
\hline \multirow{2}{*}{ Relations with patients } & Satisfactory/very satisfactory & 339 & 26.0 & $1^{* * *}$ & \multirow[b]{2}{*}{$1.29-2.04$} & 66 & 22.1 & $1^{* *}$ & \multirow[b]{2}{*}{$1.38-3.04$} \\
\hline & Moderately satisfactory/unsatisfactory & 52 & 42.3 & 1.63 & & 19 & 45.2 & 2.05 & \\
\hline \multirow{2}{*}{ Relations with families } & Satisfactory/very satisfactory & 287 & 25.5 & $1^{* * * *}$ & \multirow[b]{2}{*}{$1.30-1.95$} & 58 & 24.1 & 1 & \multirow[b]{2}{*}{$0.79-1.74$} \\
\hline & Moderately satisfactory/unsatisfactory & 76 & 40.6 & 1.59 & & 27 & 28.4 & 1.18 & \\
\hline \multirow{2}{*}{ Relations with management } & Satisfactory/very satisfactory & 231 & 22.4 & $1^{* * * *}$ & \multirow[b]{2}{*}{$1.52-2.12$} & 52 & 22.4 & 1 & \multirow[b]{2}{*}{$0.94-1.98$} \\
\hline & Moderately satisfactory/unsatisfactory & 153 & 40.4 & 1.80 & & 33 & 30.6 & 1.36 & \\
\hline \multirow[b]{2}{*}{ Effort/reward imbalance } & No & 317 & 25.0 & $1^{* * * *}$ & \multirow[b]{2}{*}{$1.59-2.34$} & 72 & 23.2 & $1^{*}$ & \multirow[b]{2}{*}{$1.18-2.95$} \\
\hline & Yes & 70 & 48.3 & 1.93 & & 13 & 43.3 & 1.87 & \\
\hline \multirow{2}{*}{ Overcommitment } & No & 268 & 25.2 & $1^{* *}$ & \multirow[b]{2}{*}{$1.12-1.60$} & 48 & 21.0 & $1^{*}$ & \\
\hline & Yes & 123 & 33.7 & 1. 34 & & 37 & 32.7 & 1.56 & $1.08-2.25$ \\
\hline & No & 243 & 22.6 & $1^{* * * *}$ & & 49 & 22.6 & 1 & \\
\hline Extrinsic effort & Yes & 148 & 41.8 & 1.85 & $1.57-2.18$ & 36 & 28.8 & 1.27 & $0.88-1.85$ \\
\hline & Yes & 234 & 22.3 & $1^{* * * *}$ & & 60 & 22.2 & $1^{*}$ & \\
\hline Reward & No & 157 & 41.6 & 1.87 & $1.59-2.20$ & 25 & 34.7 & 1.56 & $1.06-2.30$ \\
\hline Earnings & Yes & 151 & 20.8 & $1^{* * * *}$ & & 45 & 20.9 & $1^{*}$ & \\
\hline Earnings & No & 226 & 35.8 & 1.72 & $1.44-2.05$ & 37 & 33.0 & 1.57 & $1.09-2.28$ \\
\hline & Yes & 174 & 20.9 & $1^{* * * *}$ & & 41 & 20.3 & $1^{* *}$ & \\
\hline Esteem & No & 202 & 37.6 & 1.80 & $1.51-2.13$ & 43 & 35.6 & 1.65 & $1.15-2.39$ \\
\hline & Yes & 128 & 19.1 & $1^{* * * *}$ & & 31 & 16.6 & $1^{* * * *}$ & \\
\hline Job security & No & 258 & 35.9 & 1.89 & $1.57-2.26$ & 54 & 35.8 & 2.15 & $1.46-3.17$ \\
\hline Medical Factors & & $\mathbf{N}$ & $\%$ & PR & {$[95 \% \mathrm{CI}]$} & $\mathbf{N}$ & $\%$ & PR & {$[95 \% \mathrm{CI}]$} \\
\hline Psychologicaldistress & No & 200 & 37.8 & $1^{* * * *}$ & & 45 & 31.5 & $1 *$ & \\
\hline Psycnological aistress & Yes & 189 & 21.5 & 1.76 & $1.49-2.08$ & 39 & 19.7 & 1.59 & $1.10-2.32$ \\
\hline Sninal complaints & No & 98 & 19.6 & $1^{* * *}$ & & 22 & 17.9 & $1 *$ & \\
\hline spinal compraints & Yes & 293 & 31.7 & 1.62 & $1.32-1.98$ & 61 & 28.2 & 1.58 & $1.02-2.44$ \\
\hline Unner-limb complaints & No & 303 & 26.2 & $1^{*}$ & & 63 & 22.9 & $1^{\nabla}$ & \\
\hline Upper-limb complaints & Yes & 85 & 33.5 & 1.28 & $1.05-1.56$ & 21 & 33.3 & 1.45 & $0.96-2.19$ \\
\hline Lower-limb complaints. & No & 311 & 25.8 & $1^{* * *}$ & & 64 & 22.8 & $1^{*}$ & \\
\hline Lower-limb complaints & Yes & 77 & 37.6 & 1.45 & $1.19-1.78$ & 21 & 36.8 & 1.62 & $1.08-2.42$ \\
\hline
\end{tabular}

PR: Prevalence Ratio; CI: Confidence Interval. ${ }^{\nabla} p$-value $<0.1 ;{ }^{*} p<0.05 ;{ }^{* *} p<0.01 ;{ }^{* * *} p<0.001 ;{ }^{* * * *} p \leq 10^{-4}$. 
Psychosocial factors: In both nursing assistants and registered nurses, intention to leave was significantly associated with impaired relations with residents, poor reward in terms of earnings, job security, and esteem, over-commitment, and ERI.

In nursing assistants, intention to leave was significantly associated with physical, or verbal aggression, and impaired relations with management, or families.

In registered nurses, intention to leave was significantly associated with impaired relations with the care-team (Table 1).

Med Psychosocial factors: In both nursing assistants and registered nurses, intention to leave was significantly associated with impaired relations with residents, poor reward in terms of earnings, job security, and esteem, over-commitment, and ERI.

In nursing assistants, intention to leave was significantly associated with physical, or verbal aggression, and impaired relations with management, or families.

In registered nurses, intention to leave was significantly associated with impaired relations with the care-team (Table 1).

ical factors: For both nursing assistants and registered nurses, psychological distress and spinal, or lower-limb complaints were significant. Upper-limb musculoskeletal disorder was significantly associated with intention to leave in nursing assistants (Table 1).

\subsection{Factors Associated with Intention to Leave Work on Multivariate Analysis}

Occupational factors: Severe hardship related to proximity to death was associated with intention to leave in registered nurses, as was severe hardship related to residents' intellectual deterioration and weak attachment to residents in nursing assistants (Tables 2-4). In this global multivariate analysis, hardship related to inadequate equipment no longer featured as a significant factor (Table 4).

Psychosocial factors: Impaired relations with management in nursing assistants, impaired relations with residents or with care team in registered nurses, remained associated with intention to leave. In nursing assistants, high levels of effort and poor reward in terms of job security were associated with intention to leave. Poor esteem from colleagues no longer featured as a significant factor on global multivariate analysis (Tables 2 and 4).

Medical factors: Lower limb complaints and spinal complaints remained associated with intention to leave in nursing assistants but not registered nurses on global multivariate analysis (Table 4).

Table 2. Significant associations between nursing assistants' intention to leave work with the elderly and sociodemographic, occupational, psychosocial and medical factors on intermediate multivariate analysis.

\begin{tabular}{lccc}
\hline $\begin{array}{l}\text { Variables } \\
\text { Sociodemographic Factors }\end{array}$ & PR & [95\% CI] \\
\hline \multirow{2}{*}{ Age } & $<30$ years & $1^{* * *}$ & \\
& $30-39$ years & 0.91 & $0.74-1.14$ \\
& $40-49$ years & 0.72 & $0.58-0.90$ \\
& $\geq 50$ years & 0.60 & $0.46-0.78$ \\
\hline Occupational Factors & & & \\
\hline Work contract & Permanent & $1^{*}$ & \\
& Temporary & 0.78 & $0.63-0.97$ \\
\hline Hardship: equipment & Mild & $1^{* *}$ & \\
& Moderate & 1.23 & $1.00-1.51$ \\
& Severe & 1.45 & $1.17-1.79$ \\
\hline Hardship: residents & Mild & $1^{* * * *}$ & \\
intellectual deterioration & Moderate & 1.41 & $1.05-1.89$ \\
& Severe & 1.94 & $1.48-2.55$ \\
\hline \multirow{2}{*}{$\begin{array}{c}\text { Do you get attached to } \\
\text { the residents? }\end{array}$} & Very often & $1^{* *}$ & \\
& Often & 1.29 & $1.00-1.66$ \\
& Seldom & 1.48 & $1.11-1.97$ \\
& Rarely/never & 1.96 & $1.33-2.87$ \\
\hline
\end{tabular}


Table 2. Cont

\begin{tabular}{cccc}
\hline Variables & PR & [95\% CI] \\
\hline Psychosocial Factors & Satisfactory/very & $1 *$ & \\
\hline $\begin{array}{c}\text { Relations with } \\
\text { management } \\
\text { Modisfactory }\end{array}$ & satisfactory / unsatisfactory & 1.26 & $1.04-1.55$ \\
\hline Extrinsic effort & No & $1^{* *}$ & $1.08-1.60$ \\
\hline Esteem & Yes & 1.31 & $1.01-1.57$ \\
\hline Job security & Yes & $1^{*}$ & $1.21-1.89$ \\
\hline Nedical Factors & Yes & $1^{* * *}$ & 1.51 \\
\hline Psychological distress & No & 1.26 & $1.31-1.84$ \\
\hline Spinal complaints & No & 1.55 & $1.13-1.70$ \\
\hline Lower-limb complaints & Yes & $1^{* * *}$ & 1.38 \\
\hline
\end{tabular}

PR: Prevalence Ratio; CI: Confidence Interval. ${ }^{*} p<0.05 ;{ }^{* *} p<0.01 ;{ }^{* * *} p<0.001 ;{ }^{* * * *} p \leq 10^{-4}$.

Table 3. Significant associations between registered nurses' intention to leave work with the elderly and sociodemographic, occupational, psychosocial and medical factors on intermediate multivariate analysis.

\begin{tabular}{|c|c|c|c|}
\hline Variables & & PR & {$[95 \% \mathrm{CI}]$} \\
\hline \multicolumn{4}{|l|}{ Sociodemographic Factors } \\
\hline \multirow{4}{*}{ Age } & $<30$ years & $1 *$ & \\
\hline & $30-39$ years & 1.20 & $0.71-2.01$ \\
\hline & 40-49 years & 0.93 & $0.54-1.57$ \\
\hline & $\geq 50$ years & 0.62 & $0.35-1.10$ \\
\hline \multicolumn{4}{|l|}{ Occupational Factors } \\
\hline \multirow{3}{*}{ Hardship: equipment } & Mild & $1 *$ & \\
\hline & Moderate & 1.25 & $0.80-1.95$ \\
\hline & Severe & 1.75 & $1.14-2.68$ \\
\hline \multirow{3}{*}{$\begin{array}{c}\text { Hardship: proximity to } \\
\text { death }\end{array}$} & Mild & $1^{* * *}$ & \\
\hline & Moderate & 1.17 & $0.73-1.89$ \\
\hline & Severe & 1.95 & $1.23-3.08$ \\
\hline \multicolumn{4}{|l|}{ Psychosocial Factors } \\
\hline \multirow[t]{2}{*}{ Relations with team } & $\begin{array}{c}\text { Satisfactory/very } \\
\text { satisfactory }\end{array}$ & $1^{* *}$ & \\
\hline & $\begin{array}{c}\text { Moderately } \\
\text { satisfactory/unsatisfactory }\end{array}$ & 1.98 & $1.32-2.98$ \\
\hline \multirow[t]{2}{*}{ Relations with patients } & $\begin{array}{c}\text { Satisfactory/very } \\
\text { satisfactory }\end{array}$ & $1 *$ & \\
\hline & $\begin{array}{c}\text { Moderately } \\
\text { satisfactory/unsatisfactory }\end{array}$ & 1.43 & $0.93-2.19$ \\
\hline \multicolumn{4}{|l|}{ Medical Factors } \\
\hline \multirow{2}{*}{ Psychological distress } & No & $1 * *$ & \\
\hline & Yes & 1.66 & $1.14-2.39$ \\
\hline \multirow{2}{*}{ Spinal complaints } & No & $1 *$ & \\
\hline & Yes & 1.65 & $1.07-2.53$ \\
\hline
\end{tabular}

PR: Prevalence Ratio; CI: Confidence Interval. ${ }^{*} p<0.05 ;{ }^{* *} p<0.01 ;{ }^{* * *} p<0.001$. 
Table 4. Significant associations between registered nurses' and nursing assistants' intention to leave work with the elderly and sociodemographic, occupational, psychosocial and medical factors on global multivariate analysis.

\begin{tabular}{|c|c|c|c|c|c|c|c|}
\hline \multicolumn{4}{|c|}{ Nursing Assistants } & \multicolumn{4}{|c|}{ Registered Nurses } \\
\hline Variables & & PR & {$[95 \% \mathrm{CI}]$} & \multicolumn{2}{|r|}{ Variables } & PR & {$[95 \% \mathrm{CI}]$} \\
\hline Sociodemographic Factors & & & & \multicolumn{4}{|c|}{ Sociodemographic Factors } \\
\hline \multirow{4}{*}{ Age } & $<30$ years & $1^{* * *}$ & & \multirow{4}{*}{ Age } & $<30$ years & $1 *$ & \\
\hline & $30-40$ years & 0.92 & $0.75-1.12$ & & $30-40$ years & 0.97 & $0.60-1.57$ \\
\hline & $40-50$ years & 0.74 & $0.61-0.90$ & & $40-50$ years & 0.80 & $0.49-1.33$ \\
\hline & $>50$ years & 0.58 & $0.46-0.74$ & & $>50$ years & 0.53 & $0.30-0.92$ \\
\hline Occupational Factors & & & & \multicolumn{4}{|c|}{ Occupational Factors } \\
\hline \multirow{4}{*}{$\begin{array}{l}\text { Hardship: residents' } \\
\text { intellectual deterioration }\end{array}$} & Mild & $1^{* * * *}$ & & \multirow{3}{*}{$\begin{array}{l}\text { Hardship: } \\
\text { proximity to death }\end{array}$} & Mild & $1 *$ & \\
\hline & Moderate & 1.38 & $1.04-1.83$ & & Moderate & 1.23 & $0.79-1.93$ \\
\hline & Severe & 1.77 & $1.36-2.30$ & & Severe & 1.82 & $1.18-2.82$ \\
\hline & & & & \multicolumn{4}{|c|}{ Psychosocial Factors } \\
\hline \multirow{4}{*}{$\begin{array}{l}\text { Do you get attached } \\
\text { to the residents? }\end{array}$} & Very often & $1^{* *}$ & & \multirow{2}{*}{$\begin{array}{l}\text { Relations with } \\
\text { team }\end{array}$} & Satisfactory/very satisfactory & $1 * *$ & \\
\hline & Often & 1.28 & $1.01-1.63$ & & Moderately satisfactory/unsatisfactory & 1.71 & $1.15-2.56$ \\
\hline & Seldom & 1.33 & $1.02-1.73$ & Relations with & Satisfactory/very satisfactory & $1 *$ & \\
\hline & Rarely/never & 1.96 & $1.63-2.82$ & patients & Moderately satisfactory/unsatisfactory & 1.52 & $1.02-2.26$ \\
\hline \multicolumn{4}{|l|}{ Psychosocial Factors } & & & & \\
\hline \multirow{2}{*}{ Relations with management } & Satisfactory/very satisfactory & $1 * * *$ & & & & & \\
\hline & Moderately satisfactory/unsatisfactory & 1.34 & $1.14-1.58$ & & & & \\
\hline \multirow[b]{2}{*}{ Extrinsic effort } & No & $1^{*}$ & & & & & \\
\hline & Yes & 1.22 & $1.02-1.45$ & & & & \\
\hline \multirow{2}{*}{ Job security } & Yes & $1 * * *$ & & & & & \\
\hline & No & 1.42 & $1.17-1.72$ & & & & \\
\hline \multicolumn{8}{|l|}{ Medical Factors } \\
\hline \multirow{2}{*}{ Spinal complaints } & No & $1^{*}$ & & & & & \\
\hline & Yes & 1.27 & $1.04-1.55$ & & & & \\
\hline \multirow{2}{*}{ Lower-limb complaints } & No & $1 * *$ & & & & & \\
\hline & Yes & 1.34 & $1.13-1.59$ & & & & \\
\hline
\end{tabular}




\section{Discussion}

In the present study, care-workers' intention to leave was significantly related to certain psychosocial factors and impaired health status. This study of 1770 female registered nurses and nursing assistants in 105 nursing homes for the elderly in the Rhône-Alpes Region of France had a $98 \%$ response rate. This study has the advantage of having exploited for the administration of the questionnaires the periodic visits conducted by the occupational physicians. This method usually allows a high participation and minimizes the possibility that the answers are influenced by other workers, because the time in the waiting room is very limited [27].

Validated questionnaires assessed psychosocial demand, musculoskeletal complaints and psychological distress. The interest of the study lay in correlating intention to leave work with the elderly and working conditions (psychosocial and organizational demand) while taking into account the registered nurses' and nursing assistants' psychological and physical health status. This cross-sectional study does not allow us to know the temporal relation between the different phenomena that influence the intention to leave. However, previous studies have made it clear that the various factors, violence and stress, are cyclically related to each other [28-30].

Four hundred and seventy-six $(26.3 \%)$ of respondents wished to leave their work with the elderly, and nursing assistants (26.8\%) more frequently than registered nurses $(24.2 \%)$. This finding agrees with Fochsen et al., who reported that "being an assistant nurse was associated with leaving nursing care" [11]. The NEXT-Study (Nurses' early exit study) was established to investigate the degree, reasons, circumstances, and consequences of premature departure from the nursing profession in Europe. The proportion of nurses $(24.9 \%)$ who had frequently considered leaving their profession in nursing homes was higher in our study than in the French data of the longitudinal NEXT-Study $(16.5 \%)$, in which nurses were working in residential care for the elderly, hospital structures or home-care [31]. The negative relation-ship between age and turnover intent has been widely reported in previous studies [13,32]. It has been argued that young nurses who belong to generation Y (born after 1980) hold different attitudes and values towards their work, which may influence their retention. According to Lavoie-Tremblay et al., the generation $\mathrm{Y}$ nurses reported that recognition was a key motivator. Their needs are stability, flexible work schedules and shifts, recognition, opportunities for professional development, and adequate supervision [33].

The study highlighted a significant association between hardship in relation to proximity to death and intention to leave work with the elderly. Previous study showed that nursing home staff who had had palliative care training were less liable to report severe hardship in relation to proximity to death, suggesting that palliative care training has positive impact on perceived hardship in relation to proximity to death [34]. Better training in the management of dementia patients and in palliative care might reduce the intention to leave work with the elderly.

A number of researchers have reported an association between workload, stress and turnover intention $[35,36]$. In this cross-sectional study, both workplace violence and bad relationships (which often fall into verbal violence) are significantly associated with the intention to leave work. The effect was higher in younger workers; this confirms what observed in previous Italian cohorts of nurse and nursing students [37]. Previous studies suggested that the psychologically strenuous and stressful nature of the work causes nurses to consider leaving the nursing profession [38-40]. In the present study, intention to leave was significantly related to certain psychosocial factors. First effort/reward imbalance was significantly related to intention to leave in nursing assistants. The ERI model was used to analyze intention to leave the nursing profession in nurses $(n=21,299)$ in seven European countries, in the NEXT-Study: ERI was associated with the frequency of considering leaving the nursing profession [31]. Secondly, overcommitment was not significantly associated with intention to leave in the present study, in agreement with previous findings [31,40]. According to Zeytinoglu et al., nurses with higher levels of career commitment showed statistically significantly lower levels of propensity to leave nursing [10]. Wang et al. found that a higher level of occupational commitment was related to stronger intention to stay [41]. Low affective commitment to the profession was 
associated with greater intention to leave the profession [2]. Moreover, Takase et al. found that nursing staff commitment was directly related to reduce intention to quit the profession [42]. The present study highlighted weak attachment to residents on the part of nurses and nursing assistants as associated with intention to leave. Then high levels of effort were an important work environment factor for intention to leave, consistent with previous findings [31-40]. Finally, for nursing assistants, poor reward in terms of job security, but in terms of not earnings, was significantly associated with intention to leave on multivariate analysis. Pay was found not a significant predictor for Jordanian nurses to leave their organization [43]. According to Lavoie-Tremblay et al., the reasons most often given for quitting the profession by nurses were difficult working conditions and a lack of job stability [34]. Improving the psychosocial work environment and specifically occupational rewards may be helpful in retaining caregivers.

Poor relations with management for nursing assistants and poor relations with the care-team and patients for nurses were significantly associated with intention to leave [44]. Nursing assistants seemed to exchange little with nurses according to an ergonomic study of geriatric care-staff [45]. In France, the working week was reduced to $35 \mathrm{~h}$, which may have had the effect of reducing the time available for verbal exchanges within the team. Moreover, increasing administrative work for nurses and the increased work-load reported may have reduced the time available for verbal exchanges between patients and nurses, impairing the nurse-patient relationship. Cowden et al., in a systematic review of the literature, found a positive relationship between transformational leadership, supportive work environments and staff nurses intentions to stay [46]. Creating a supportive work environment, with supportive leadership gives nurses the confidence to stay $[47,48]$. Healthy and supportive work relationships have been shown to be related to greater intention to stay in work for nurses $[49,50]$.

The present study revealed a significant association between intention to leave and impaired health status. First, lower-limb and spinal musculoskeletal complaints were significantly associated with intention to leave for nursing assistants. Fochsen and Sjögren showed that musculoskeletal problems of the neck/shoulder and knees were associated with leaving nursing care [11,51]. Secondly, psychological distress was significantly associated with intention to leave for both nursing assistants and nurses, in line with the longitudinal study of 6441 caregivers by Kivimaki et al. [52]. Bartram et al. found that emotional labor has an indirect effect on intention to leave via burnout nurses experience [53].

\section{Conclusions}

Care-staff in nursing homes for the elderly are exposed to elevated psychological and physical demands in caring for dependent elderly persons often with comorbidities. The present study found that some of the demands experienced by nurses and nursing assistants underlie an intention to leave work with the elderly. The results highlight the importance of perceived working conditions in this intention to leave. Policy to reduce voluntary turnover in nursing homes for the elderly could be based on a multifactorial approach involving work organization, reduced psychosocial demand and access to certain training modules such as in palliative care and the management of patients in dementia. Reorganization to reduce nurses' administrative workload by delegating tasks to administration workers should allow nurses more time for exchanges with both patients and nursing assistants. Having more time to devote to relational aspects would enhance verbal exchange between residents and caregivers, which in turn should improve job satisfaction and quality of care. Greater involvement in drawing up personalized care projects and reinforcing and developing caregiver autonomy in performing everyday care tasks should improve the caregivers' image of the job. Better recognition of the work accomplished could be achieved by increasing the time available for exchanges between colleagues and with management, and by improving job security.

Author Contributions: Conceptualization, B.C. and L.F.; Data curation, C.P., E.F. and B.C.; Formal analysis, C.P. and E.F.; Funding acquisition, B.C.; Investigation, C.P. and B.C.; Methodology, C.P., B.C., J.B.F., E.F. and L.F.; Project 
administration, B.C. and J.B.F.; Supervision, B.C. and L.F.; Validation, C.P., B.C., E.F., J.B.F. and L.F.; Visualization, C.P., J.B.F., E.F. and L.F.; Writing-original draft, C.P.

Funding: This research was funded by [Department of Businesses, Competition, Consumption, Work and Employment (DIRECCTE)].

Acknowledgments: The authors thank all of the occupational physicians who agreed to assist with this study.

Conflicts of Interest: The authors declare no conflict of interest.

\section{References}

1. Kovner, C.T.; Brewer, C.S.; Fatehi, F.; Jun, J. What does nurse turnover rate mean and what is the rate? Policy Politics Nurs. Pract. 2014, 15, 64-71. [CrossRef] [PubMed]

2. Flinkman, M.; Leino-Kilpi, H.; Salanterä, S. Nurses' intention to leave the profession: Integrative review. J. Adv. Nurs. 2010, 66, 1422-1434. [CrossRef] [PubMed]

3. Brannon, D.; Zinn, J.S.; Mor, V.; Davis, J. An Exploration of Job, Organizational, and Environmental Factors Associated with High and Low Nursing Assistant Turnover. Gerontologist 2002, 42, 159-168. [CrossRef] [PubMed]

4. Mukamel, D.B.; Spector, W.D.; Limcangco, R.; Wang, Y.; Feng, Z.; Mor, V. The costs of turnover in nursing homes. Med. Care 2009, 47, 1039. [CrossRef] [PubMed]

5. Cohen-Mansfield, J. Turnover among nursing home staff. A review. Nurs. Manag. 1997, 28, 59-62, 64. [CrossRef]

6. Pelissier, C.; Fontana, L.; Fort, E.; Agard, J.P.; Couprie, F.; Delaygue, B.; Glerant, V.; Perrier, C.; Sellier, B.; Vohito, M.; et al. Occupational risk factors for upper-limb and neck musculoskeletal disorder among health-care staff in nursing homes for the elderly in France. Ind. Health 2014, 52, 334-346. [CrossRef] [PubMed]

7. Moss, A.J.; Harris-Kojetin, L.D.; Sengupta, M.; Park-Lee, E.; Caffrey, C.; Rosenoff, E.; Wiener, J.M.; Greene, A.M. Design and Operation of the 2010 National Survey of Residential Care Facilities. Vital Health Stat Ser. 1 Programs Collect Proced. 2011, 54, 1-131.

8. Spidla, V. Long Term Care in the European Union; European Commission: Brussels, Belgium, 2008.

9. Pélissier, C.; Vohito, M.; Fort, E.; Sellier, B.; Agard, J.P.; Fontana, L.; Charbotel, B. Risk factors for work-related stress and subjective hardship in health-care staff in nursing homes for the elderly: A cross-sectional study. J. Occup. Health 2015, 57, 285-296. [CrossRef] [PubMed]

10. Zeytinoglu, I.U.; Denton, M.; Davies, S.; Baumann, A.; Blythe, J.; Boos, L. Retaining nurses in their employing hospitals and in the profession: Effects of job preference, unpaid overtime, importance of earnings and stress. Health Policy 2006, 79, 57-72. [CrossRef] [PubMed]

11. Fochsen, G.; Josephson, M.; Hagberg, M.; Toomingas, A.; Lagerström, M. Predictors of leaving nursing care: A longitudinal study among Swedish nursing personnel. Occup. Environ. Med. 2006, 63, 198-201. [CrossRef] [PubMed]

12. Pélissier, C.; Fontana, L.; Fort, E.; Vohito, M.; Sellier, B.; Perrier, C.; Glerant, V.; Couprie, F.; Agard, J.P.; Charbotel, B. Impaired mental well-being and psychosocial risk: A cross-sectional study in female nursing home direct staff. BMJ Open 2015, 5, e007190. [CrossRef] [PubMed]

13. Chiang, Y.-M.; Chang, Y. Stress, depression, and intention to leave among nurses in different medical units: Implications for healthcare management/nursing practice. Health Policy 2012, 108, 149-157. [CrossRef] [PubMed]

14. Jolivet, A.; Caroly, S.; Ehlinger, V.; Kelly-Irving, M.; Delpierre, C.; Balducci, F.; Sobaszek, A.; De Gaudemaris, R.; Lang, T. Linking hospital workers' organisational work environment to depressive symptoms: A mediating effect of effort-reward imbalance? The ORSOSA study. Soc. Sci. Med. 2010, 71, 534-540. [CrossRef] [PubMed]

15. Van Vegchel, N.; de Jonge, J.; Bosma, H.; Schaufeli, W. Reviewing the effort-reward imbalance model: Drawing up the balance of 45 empirical studies. Soc. Sci. Med. 2005, 60, 1117-1131. [CrossRef] [PubMed]

16. Siegrist, J.; Starke, D.; Chandola, T.; Godin, I.; Marmot, M.; Niedhammer, I.; Peter, R. The measurement of effort-reward imbalance at work: European comparisons. Soc. Sci. Med. 2004, 58, 1483-1499. [CrossRef] 
17. Niedhammer, I.; Siegrist, J.; Landre, M.F.; Goldberg, M.; Leclerc, A. Psychometric properties of the French version of the Effort-Reward Imbalance model. Revue d'Epidemiologie et de Sante Publique 2000, 48, 419-437. [PubMed]

18. Niedhammer, I.; Siegrist, J. Psychosocial factors at work and cardiovascular diseases: Contribution of the Effort-Reward Imbalance model. Revue d'Epidemiologie et de Sante Publique 1998, 46, 398-410. [PubMed]

19. Kuorinka, I.; Jonsson, B.; Kilbom, A.; Vinterberg, H.; Biering-Sørensen, F.; Andersson, G.; Jørgensen, K. Standardised Nordic questionnaires for the analysis of musculoskeletal symptoms. Appl. Ergon. 1987, 18, 233-237. [CrossRef]

20. Lesage, F.-X. Validation of the General Health Questionnaire (GHQ-12) adapted to a work-related context. Open J. Prev. Med. 2011, 1, 44-48. [CrossRef]

21. Bouletreau, A.; Chouanière, D.; Wild, P.; Fontana, J. Concevoir, traduire et valider un quetsionnaire à propos d'un exemple EUROQUEST. INRS 2009, 178, 1-49. (In French)

22. Estryn-Behar, M.; Kaminski, M.; Peigne, E.; Bonnet, N.; Vaichere, E.; Gozlan, C.; Azoulay, S.; Giorgi, M. Stress at work and mental health status among female hospital workers. Br. J. Ind. Med. 1990, 47, 20-28. [CrossRef] [PubMed]

23. Charbotel, B.; Croidieu, S.; Vohito, M.; Guerin, A.C.; Renaud, L.; Jaussaud, J.; Bourboul, C.; Imbard, I.; Ardiet, D.; Bergeret, A. Working conditions in call-centers, the impact on employee health: A transversal study. Part II. Int. Arch. Occup. Environ. Health 2009, 82, 747-756. [CrossRef] [PubMed]

24. Niedhammer, I.; Chea, M. Psychosocial factors at work and self reported health: Comparative results of cross sectional and prospective analyses of the French GAZEL cohort. Occup. Environ. Med. 2003, 60, 509-515. [CrossRef] [PubMed]

25. Langevin, V.; François, M.; Boini, S.; Riou, A. Déséquilibre efforts/récompense. Doc. Pour. Médecin. Trav. 2011, 125, 111-115.

26. Deddens, J.; Petersen, M.; Lei, X. Estimation of prevalence ratos when PROC GENMOD does not converge. In Proceedings of the 28th Annual SAS Users Group International Conference, Washington, DC, USA, 30 March-2 April 2003; Seattle Wash SAS Institute: Cary, NC, USA, 2003.

27. Magnavita, N.; Garbarino, S. Social psychiatry in the waiting room. What a physician can learn about occupational stress from workers waiting to be examined. Psychiatry J. 2013, 2013, 701872. [CrossRef] [PubMed]

28. Magnavita, N. The exploding spark. Workplace violence in an infectious disease hospital—A longitudinal study. BMRI BioMed Res. Int. 2013, 2013, 316358. [CrossRef] [PubMed]

29. Magnavita, N. Workplace violence and occupational stress in health care workers: A chicken and egg situation-Results of a 6-year follow-up study. J. Nurs. Scholarsh. 2014, 46, 366-376. [CrossRef] [PubMed]

30. Halter, M.; Boiko, O.; Pelone, F.; Beighton, C.; Harris, R.; Gale, J.; Gourlay, S.; Drennan, V. The determinants and consequences of adult nursing staff turnover: A systematic review of systematic reviews. BMC Health Serv. Res. 2017, 17, 824. [CrossRef] [PubMed]

31. Hasselhorn, H.-M.; Tackenberg, P.; Peter, R. Effort-Reward Imbalance among Nurses in Stable Countries and in Countries in Transition. Int. J. Occup. Environ. Health 2004, 10, 401-408. [CrossRef] [PubMed]

32. Ramoo, V.; Abdullah, K.L.; Piaw, C.Y. The relationship between job satisfaction and intention to leave current employment among registered nurses in a teaching hospital. J. Clin. Nurs. 2013, 22, 3141-3152. [CrossRef] [PubMed]

33. Lavoie-Tremblay, M.; O’brien-Pallas, L.; Gélinas, C.; Desforges, N.; Marchionni, C. Addressing the turnover issue among new nurses from a generational viewpoint. J. Nurs. Manag. 2008, 16, 724-733. [CrossRef] [PubMed]

34. Pélissier, C.; Fontana, L.; Fort, E.; Charbotel, B. Pénibilité ressentie et accès aux formations chez le personnel féminin travaillant auprès des résidents dans les maisons de retraite médicalisées: Une enquête transversale. Gériatrie Psychol. Neuropsychiatr. Vieil. 2015, 13, 396-406.

35. Brannon, D.; Barry, T.; Kemper, P.; Schreiner, A.; Vasey, J. Job Perceptions and Intent to Leave Among Direct Care Workers: Evidence from the Better Jobs Better Care Demonstrations. Gerontologist 2007, 47, 820-829. [CrossRef] [PubMed]

36. Liu, Y.; Wu, L.-M.; Chou, P.-L.; Chen, M.-H.; Yang, L.-C.; Hsu, H.-T. The Influence of Work-Related Fatigue, Work Conditions, and Personal Characteristics on Intent to Leave Among New Nurses. J. Nurs. Scholarsh. 2016, 48, 66-73. [CrossRef] [PubMed] 
37. Magnavita, N.; Heponiemi, T. Workplace violence against nursing students and nurses. An Italian experience. J. Nurs. Scholarsh. 2011, 43, 203-210. [CrossRef] [PubMed]

38. Cho, S.-H.; Park, M.; Jeon, S.H.; Chang, H.E.; Hong, H.-J. Average Hospital Length of Stay, Nurses' Work Demands, and Their Health and Job Outcomes. J. Nurs. Scholarsh. 2014, 46, 199-206. [CrossRef] [PubMed]

39. Li, J.; Fu, H.; Hu, Y.; Shang, L.; Wu, Y.; Kristensen, T.S.; Mueller, B.H.; Hasselhorn, H.M. Psychosocial work environment and intention to leave the nursing profession: Results from the longitudinal Chinese NEXT study. Scand. J. Public Health 2010, 38 (Suppl. 3), 69-80. [CrossRef] [PubMed]

40. Tominaga, M.T.; Miki, A. Factors associated with the intention to leave among newly graduated nurses in advanced-treatment hospitals in Japan. Jpn. J. Nurs. Sci. 2011, 8, 33-46. [CrossRef] [PubMed]

41. Wang, L.; Tao, H.; Ellenbecker, C.H.; Liu, X. Job satisfaction, occupational commitment and intent to stay among Chinese nurses: A cross-sectional questionnaire survey. J. Adv. Nurs. 2012, 68, 539-549. [CrossRef] [PubMed]

42. Takase, M.; Yamashita, N.; Oba, K. Nurses' leaving intentions: Antecedents and mediating factors. J. Adv. Nurs. 2008, 62, 295-306. [CrossRef] [PubMed]

43. Al-Hussami, M.; Darawad, M.; Saleh, A.; Hayajneh, F.A. Predicting nurses' turnover intentions by demographic characteristics, perception of health, quality of work attitudes. Int. J. Nurs. Pract. 2014, 20, 79-88. [CrossRef] [PubMed]

44. Zeytinoglu, I.U.; Denton, M.; Plenderleith, J.M. Flexible employment and nurses' intention to leave the profession: The role of support at work. Health Policy 2011, 99, 149-157. [CrossRef] [PubMed]

45. Estryn-Behar, M.; Chaumon, E.; Garcia, F.; Milanini-Magny, G.; Fry, C.; Bitot, T.; Ravache, A.E.; Deslandes, H. la souffrance au travail en gériatrie comprendre et agir. La Revue Francophone de Gériatrie et de Gérontologie 2013, 20, 6-27.

46. Cowden, T.; Cummings, G.; Profetto-Mcgrath, J. Leadership practices and staff nurses' intent to stay: A systematic review. J. Nurs. Manag. 2011, 19, 461-477. [CrossRef] [PubMed]

47. Kaewboonchoo, O.; Yingyuad, B.; Rawiworrakul, T.; Jinayon, A. Job stress and intent to stay at work among registered female nurses working in Thai hospitals. J. Occup. Health 2014, 56, 93-99. [CrossRef] [PubMed]

48. Mrayyan, M.T. Differences of hospitals' organisational climates and nurses' intent to stay: Nurses' perspectives. J. Res. Nurs. 2009, 14, 465-477. [CrossRef]

49. Tourangeau, A.E.; Cranley, L.A. Nurse intention to remain employed: Understanding and strengthening determinants. J. Adv. Nurs. 2006, 55, 497-509. [CrossRef] [PubMed]

50. Tourangeau, A.E.; Cummings, G.; Cranley, L.A.; Ferron, E.M.; Harvey, S. Determinants of hospital nurse intention to remain employed: Broadening our understanding. J. Adv. Nurs. 2010, 66, 22-32. [CrossRef] [PubMed]

51. Sjögren, K.; Fochsen, G.; Josephson, M.; Lagerström, M. Reasons for leaving nursing care and improvements needed for considering a return: A study among Swedish nursing personnel. Int. J. Nurs. Stud. 2005, 42, 751-758. [CrossRef] [PubMed]

52. Kivimäki, M.; Vanhala, A.; Pentti, J.; Länsisalmi, H.; Virtanen, M.; Elovainio, M.; Vahtera, J. Team climate, intention to leave and turnover among hospital employees: Prospective cohort study. BMC Health Serv. Res. 2007, 7, 170. [CrossRef] [PubMed]

53. Bartram, T.; Casimir, G.; Djurkovic, N.; Leggat, S.G.; Stanton, P. Do perceived high performance work systems influence the relationship between emotional labour, burnout and intention to leave? A study of Australian nurses. J. Adv. Nurs. 2012, 68, 1567-1578. [CrossRef] [PubMed]

(C) 2018 by the authors. Licensee MDPI, Basel, Switzerland. This article is an open access article distributed under the terms and conditions of the Creative Commons Attribution (CC BY) license (http:/ / creativecommons.org/licenses/by/4.0/). 